\begin{tabular}{|c|c|c|}
\hline & $\begin{array}{c}\text { Kalpa Publications in Engineering } \\
\text { Volume 3, 2020, Pages 36-41 }\end{array}$ & kalpa \\
\hline & $\begin{array}{l}\text { Proceedings of International Sym- } \\
\text { posium on Applied Science } 2019\end{array}$ & Engineering \\
\hline
\end{tabular}

\title{
The Application Of Penh Simulation On Proton Imaging
}

\author{
Anh Duy Nguyen ${ }^{1, *}$, Thi Kim Lan Tran ${ }^{2}$, Anh Tu Ly ${ }^{2}$ \\ ${ }^{1}$ Institute of Biomedical Engineering, National Cheng Kung University \\ ${ }^{2}$ Ho Chi Minh City University of Technology-VNUHCM \\ ng.anhduy89@gmail.com
}

\begin{abstract}
Proton therapy is one of the most accurate forms of cancer therapies, which requires accurate knowledge of the dose delivered to the patient and verification of the correct patient position with respect to the proton beam to avoid damage to critical normal tissues and geographical tumour misses. In existing proton treatment centres, dose calculation is performed based on xray computed tomography (CT) and the patient is positioned with $\mathrm{x}$-ray radiographs. The use of xray $\mathrm{CT}$ images for proton treatment planning ignores fundamental differences in physical interaction processes between photons and protons and is therefore inherently inaccurate. Further, xray radiographs depict only skeletal structures; they do not show the tumour itself. Ideally, one would image the patient directly with proton CT by measuring the energy loss of high-energy protons that traverse the patient. The main content of this report is the application of simulation program PENH proton transmission via phantom associated with the PENEASY creation in proton imaging can be applied in proton therapy.
\end{abstract}

\section{Introduction}

The idea of proton therapy had started from the early 1940', when Wilson et al. published on proton radiation therapy compared to traditional photon radiation therapy [1]. Due to the disadvantage of strangling and spreading of electrons, the concentration of ionization of proton is more efficient. The proton beam gives the most graphically distribution at the end of the spectrum as well as the very steep slope after the peak of Bragg is the most important point to limit the influence on surrounding normal tissues. In the treatment, each beam is adjusted so that it brings the volume to irradiate a uniform dose. Therefore, with a defined direction, Bragg peak must reach the deepest point in the volume to be irradiated. In beam scanning technique, its energy and intensity can be completely controlled. The greatest advantage of proton radiotherapy compared to traditional methods is that it has less impact on surrounding healthy tissue thanks to the special dose distribution in matter [2]. In photon therapy, x-ray dose is highest at the beginning of the therapy procedure before reaches tumor tissue with lower dose. 
However, proton therapy starts from low dose and reaches tumor with highest dose. Then it stops at Bragg peak and does not harm surrounding normal tissue.
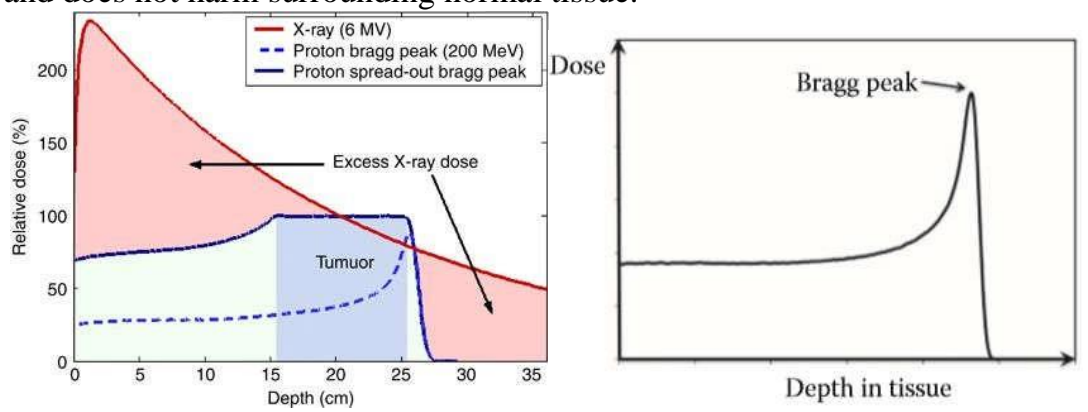

Figure 1: a. Bragg peak of Proton interaction on tissue [2], b. Radiation dose of proton therapy compared to photon therapy [3]

Proton imaging experiments over the past 50 years are based on 1) particle depletion, 2) nuclear dispersion and 3) particle monitoring in combination with energy loss. The decline of particles and energy loss settings work with no relative capacity. None of these methods has made it to clinical trials yet. Matthias et al. indicated that Proton CT slice showed bony anatomy clearly compared to X-ray CT by applying combination of the filtered backprojection algorithm and Ram-Lak filter kernel [4]. Besides that, Poludniowski et al. also recorded CT images with $62-\mathrm{MeV}$ protons on slices of a 2-cm diameter plastic test phantom with a promising spatial resolution as a fullwidth half-maximum of $0.9 \mathrm{~mm}$ [5].

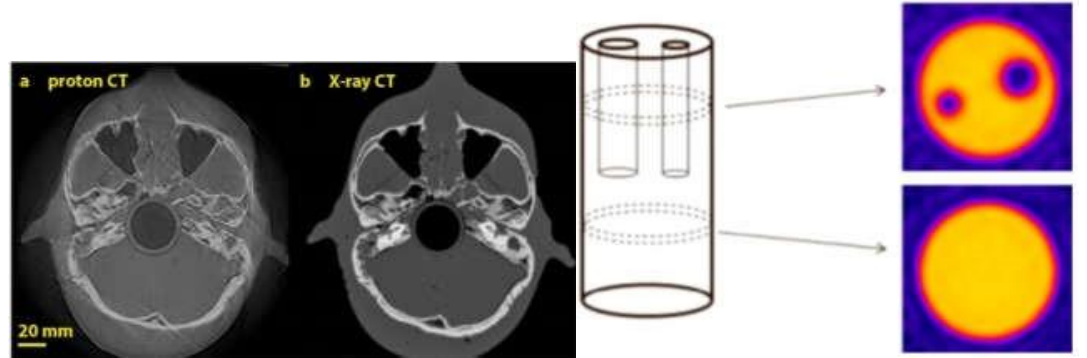

Figure 2: Proton CT and X-ray CT of bony anatomy [4] and Proton phantom [5]

In this paper, we implemented a simulation program named PENH [6] based on PENEASY [7] and PENELOPE [8] to create Proton image on a phantom with human head structure. From this simulation, we could consider the result to do further experiment as well as for proton radiation therapy in the future.

\section{Methodology}

PENH is based on mixed mechanical simulation for elastic and inelastic collisions (electromagnetic interaction). Different ways of elastic collision are calculated using eikonal approximation function with Dirac-HartreeFork-Slater atomic capacity. The section for inelastic collision is calculated based on the relative approximation Born, using the Sternheimer-Liljequist pattern of the general vibration intensity. Simulated elastic and inelastic collision simulated using interactive scattering database for $1 \mathrm{H}$ and ICRU63 data for 12C, 14N, 16O, 31P, 40Ca. Secondary proton, alphas, and deuteron are simulated as protons, with energy set to ensure proper limits.

Immediate gamma radiation can also be simulated based on user requirements. The left figure below shows PENH simulation in water phantom and dose depth distribution by the initial energy by $100 \mathrm{MeV}$ [6] and the right figure compares photon interaction and proton interaction in different energies . It can be seen that photon energy reaches $100 \%$ dose at the depth $3 \mathrm{~cm}$ and significantly decreases at the depth 
$40 \mathrm{~cm}$. In contrast, proton therapy starts with only $40 \%$ dose and increases rapidly $100 \%$ at the end of the phantom depth.
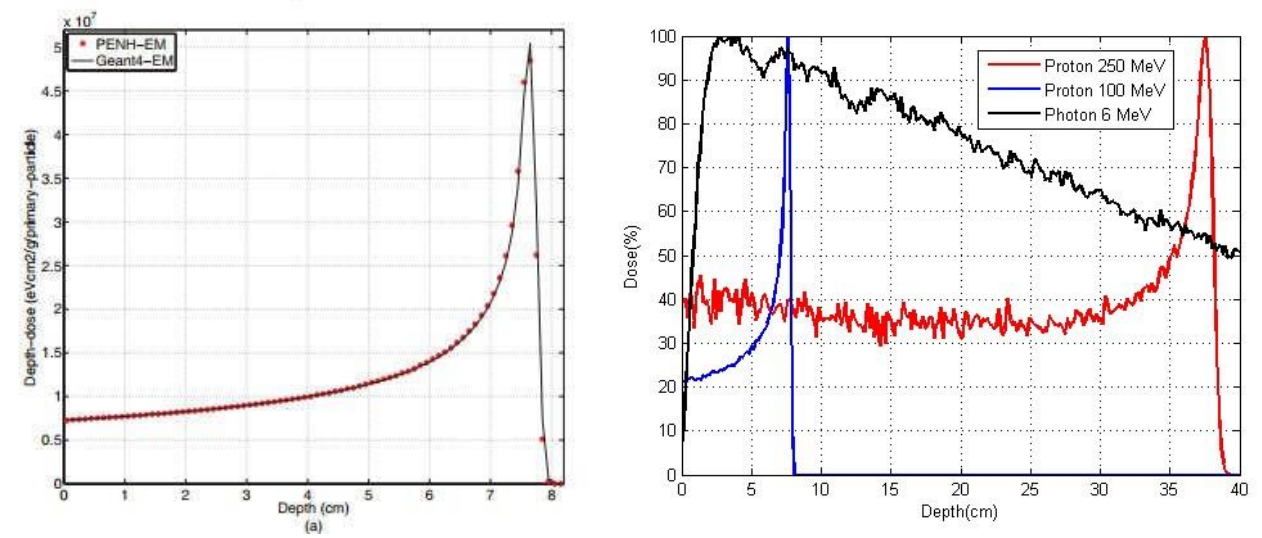

Figure 3: Nuclear scattering at $100 \mathrm{MeV}$ [6]

PENEASY [7] is an open source which supports for PENELOPE [8]. The models include a wide range of space and distributed energy: source points and Gaussian distribution sources in space, volume sources and radiation beams with arbitrary shape (quadric), differential spectrum Gaussian and continuous piece-wise spectrum, photon beam with arbitrary polarization and phase spaces.

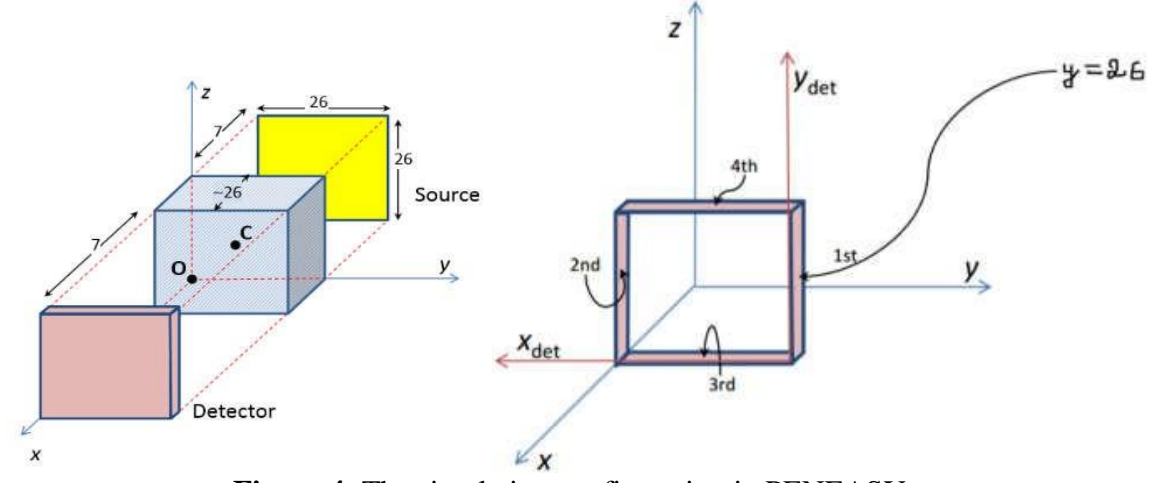

Figure 4: The simulation configuration in PENEASY

Detail steps are followed (the simulation is tested on Windows platform):

a) Create working directory on $\sim$ / penEasy /.

b) Copy all penEasy files into a folder.

c) Compile and link penEasy.f by the syntax > gfortran penEasy.f -o penEasy. $\mathrm{x}-\mathrm{O}$

d) Move penEasy.X into a executing file

e) Run penEasy following syntax

>> penEasy.x <penEasy.in> penEasy.out penEasy.in includes the simulation information such as detector parameters, radiation dose, simulation time and so on.

[SECTION SOURCE BOX ISOTROPIC GAUSS SPECTRUM v.2014-12-21] ON STATUS (ON or OFF) 4 PARTICLE TYPE (1=ELECTRON, 2=PHOTON, 3=POSITRON) SUBSECTION FOR PARTICLE POSITION: -20.0 0.0 0.0 COORDINATES (cm) OF BOX CENTER 0.0 26.0 26.0 BOX SIDES (cm) 0.0 0.0 FWHMs (cm) OF GAUSSIAN X,Y DISTRIBUTIONS 0.0 0.0 0.0 EULER ANGLES [OMEGA,THETA,PHI](deg) FOR BOX ROTATION Rz(PHI).Ry(THETA).Rz(OMEGA).r 13.013 .013 .0 TRANSLATION [DX,DY,DZ](cm) OF BOX CENTER POSITION O SOURCE MATERIAL ( $0=$ DON'T CARE, >0 FOR LOCAL SOURCE, <0 FOR IN-FIELD BEAM)

f) The simulation results are in DAT files. Gnuplot gets information form those files to plot the diagram as well as the image. 
The Proton source which has energy as $100 \mathrm{MeV}$ is emanated from a $26 \mathrm{x} 26 \mathrm{~cm} 2$ plate places at $\mathrm{x}$ $=-20 \mathrm{~cm}$, placed in the direction along the $\mathrm{x}$ axis. Phantom is built from medical images of white people (AM: male adults). Phantom AM builds on the Golem Phantom's base, developed at the German Research Centre for Environmental Health [9]. The body and mass of organs or tissues are adjusted according to basic anatomical and physiological data in ICRP 89 . The resolution of phantom, AM is $2,137 \times 2,137 \mathrm{~mm} 2$ (horizontal) $\times 8.0 \mathrm{~mm}$ (height)

\section{Result and discussion}

In figure $5 b$, it could be seen that Proton with $100 \mathrm{MeV}$ only shows the shape as human head without clear details as x-ray source. The resolution of the absorbed dose is very low and does not come to the resolution needed to be put phantom $\sim 25.64 \times \sim 25.64 \times 26.40 \mathrm{~cm} 3$ [5]. It is saved to the folder containing the files.

Array of agency identification numbers (in ASCII format); File name is: AM.dat A list of segregated structures, their identification numbers and designated vehicles;

File name is: AM_organs.dat List of environmental structures, their basic components and density; File name is: AM_media.dat

Mass ratio of bone components (eczema, red and yellow bone marrow) in spongiosa regions; File name is: AM_spongiosa.dat

Percentage of blood volume in different body tissues; File name is: AM_blood.dat

The material list in phantom VOX format: 1: vacuum (Hydro with mass density $1 \times 10-10 \mathrm{~g} / \mathrm{cm} 3$ )

2: water (PENELOPE database)

3: bone ICRP (PENELOPE database)

4: lung ICRP (PENELOPE database)

5: air (PENELOPE database)

6: spongiosa $(8.3 \% \mathrm{H}, 26.6 \% \mathrm{C}, 2.7 \% \mathrm{~N}, 51.1 \%$

$\mathrm{O}, 4.0 \% \mathrm{P}, 4.0 \% \mathrm{Ca}$, mass density: $1.228 \mathrm{~g} / \mathrm{cm} 3$ )

Automatic identification of the reference frame of the transducer (for pixel recording) requires a certain order in the definition of surface restriction. The plane $y=26$ must be the first face declared in the definition of the probe. The plane $\mathrm{y}=0$ must be the second face declared. The plane $\mathrm{z}=0$ must be the third. And the plane $\mathrm{z}=26$ must be fourth. The probe is a perfect absorber. It must be provided with a unique material ID.

into clinical practice. The causes of errors may include: 1) Personal errors during the simulation run, during the simulation process, individuals make mistakes in entering the input parameters and turn off the incorrect interactions. 2) The input file has an error. This may be the cause of resulting in low resolution results. 3) Running on the ground simulating electrons, photons and positrons. PenEasy is originally built to simulate radiation images of electrons, photons and positrons so when combining it with penH to simulate proton images, some programming errors can occur. 

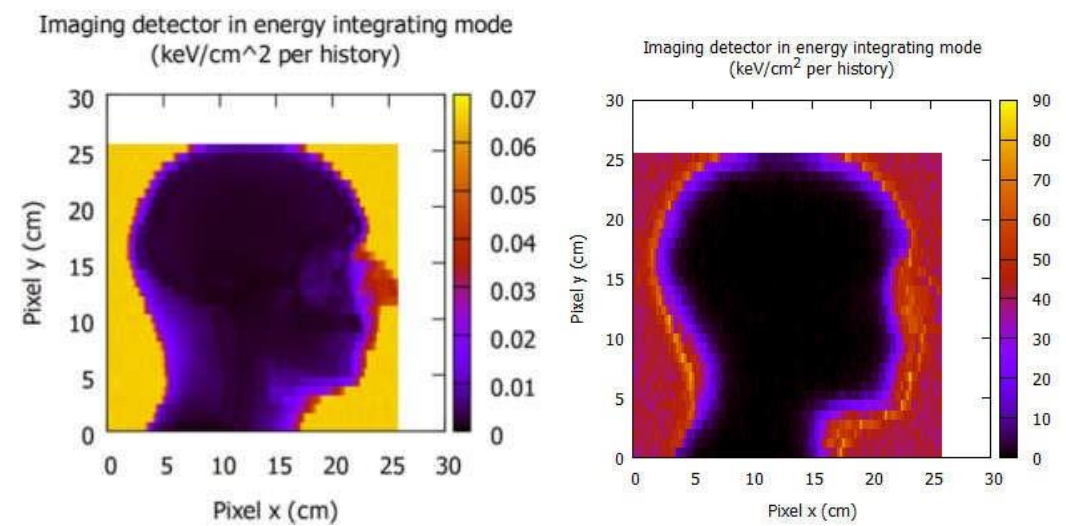

Figure 5: a. Human head phantom simulated by x-ray source [7];

b. Human head phantom simulated by proton source.

\section{Conclusion}

The number of cancer patients is increasing, and need appropriate and effective treatments. Therefore, many treatments have been implemented to effectively treat many types of cancer. Proton radiotherapy is one of the very precise forms of cancer treatment, requiring precise determination of the dose to the patient and the right position of the patient compared to the proton beam to avoid damage to healthy tissues. This method requires accurate image diagnosis, but tomography and magnetic resonance imaging cannot be brought about properly due to the calibration between the proton stopping energy and the required photon mass reduction factor. be done. Because this translation is complicated by differences in interaction mechanisms, it is necessary to increase the limit around the tumor location. Many irradiated tissue is a consequence, reducing the benefits of proton radiotherapy. This problem will be solved when protons are used for imaging purposes. When protons are dispersed significantly, they must be monitored separately before and after passing through the patient. Radiography of different density in the body is taken from the energy loss measured along the regeneration path. This method has not been widely used and is still being studied for optimal parameters such as angle of shooting, number of angles of shooting or dose. To support finding the most optimal parameters, Monte Carlo simulation programs have been used to simulate equipment. PENELOPE and PENEASY, PENH programs have simulated phantom head with tissue details at different densities. However, the simulation results also need to be improved better on parameters such as increased resolution to improve image quality. The simulation results of PENELOPE are only the references; it needs to be compared with the results of other methods as well as with practical measurement results.

\section{References}

Wilson RR, "Radiological use of fast protons”, Radiology., vol. 47, no. 5, pp. 487-491, 1946.

Efstathiou J, "Proton beam therapy and localized prostate cancer: current status and controversies", British Journal of Cancer., vol. 108, pp. 1225-1230, 2013.

Harald P. Proton therapy physics, CRC Press, U.S, 2012.

Matthias P, "High-energy proton imaging for biomedical applications", Nature Sci Rep., vol. 6, 2016. 
Poludniowski G, "Review article: Proton radiography and tomography with application to proton therapy", Br J Radiol., vol. 88, 2015.

Sterpin E, "Extension of PENELOPE to protons: simulation of nuclear reactions and benchmark with Geant4", Med. Phys., vol. 40, no. 11, pp. 111705-1 - 111705-11, 2013

Josep S, "A PENELOPE-based system for the automated Monte Carlo simulation of clinacs and voxelized geometries - application to far-from-axis fields", Med. Phys., vol. 38, no. 11, pp. 5887-5895, 2011.

Francesc S, "The PENELOPE code system. Specific features and recent improvements", presented at the Joint International Coference on Supercomputing in Nuclear Applications and Monte Carlo, Paris, 2013.

Maria Z, "Adult male and female reference computational phantoms (ICRP publication 110)", Jpn. J. Health Phys., vol. 45, no. 4, pp. 357-369, 2010. 